\title{
Quantum black hole entropy from 4d supersymmetric Cardy formula
}

\author{
Masazumi Honda* \\ Department of Applied Mathematics and Theoretical Physics, \\ Centre for Mathematical Sciences, University of Cambridge, \\ Wilberforce Road, Cambridge, CB3 OWA, United Kingdom
}

(Received 11 February 2019; revised manuscript received 2 April 2019; published 17 July 2019)

We study the canonical AdS/CFT correspondence between $4 \mathrm{~d} S U(N) \mathcal{N}=4$ super Yang-Mills theory (SYM) and type IIB superstring theory on $\mathrm{AdS}_{5} \times S^{5}$. We analyze the supersymmetric index of the $\mathcal{N}=4$ SYM on $S^{1} \times M_{3}$ which counts supersymmetric states with fixed quantum numbers. We compute an asymptotic behavior of the index in the limit of shrinking $S^{1}$ for any $N$ by a refinement of the $4 \mathrm{~d}$ supersymmetric Cardy formula. The asymptotic behavior for the superconformal index case $\left(M_{3}=S^{3}\right)$ at large $N$ agrees with the Bekenstein-Hawking entropy of a rotating electrically charged BogomolnyiPrasad-Sommerfeld (BPS) black hole in $\mathrm{AdS}_{5}$ via a Legendre transformation as recently shown in the literature. We also find that the agreement formally persists for finite $N$ if we slightly modify the AdS/CFT dictionary between the Newton constant and $N$. This implies the existence of a nonrenormalization property of the black hole entropy against quantum corrections. We also study the cases with other gauge groups and additional matter and the orbifold $\mathcal{N}=4 \mathrm{SYM}$. It turns out that the entropies of all the CFT examples in this paper are universally given by $2 \pi \sqrt{Q_{1} Q_{2}+Q_{1} Q_{3}+Q_{2} Q_{3}-2 c\left(J_{1}+J_{2}\right)}$ with charges $Q_{1,2,3}$, angular momenta $J_{1,2}$, and central charge $c$. The results for other $M_{3}$ make predictions to the gravity side.

DOI: $10.1103 /$ PhysRevD.100.026008

\section{INTRODUCTION}

It is widely expected that black hole mechanics is connected to thermodynamics and, in particular, a black hole has a macroscopic entropy given by a horizon area [1]. Since string theory is the candidate of consistent quantum gravity, string theory should give a microscopic explanation. As is well known, the seminal paper [2] by Strominger and Vafa has derived the Bekenstein-Hawking entropy of an asymptotically flat black hole by counting supersymmetric (SUSY) states in string theory.

In the context of AdS/CFT correspondence [3], the problem of black hole entropy is mapped into whether the entropy of an asymptotically AdS black hole is explained by counting states of a dual CFT. Namely, if there is a black hole with some quantum numbers on the gravity side, then the Bekenstein-Hawking entropy of the black hole should agree with the (log of) degeneracy of states with the same quantum numbers on the dual CFT side in the large- $N$ limit. Recently, there have been great steps to

"mh974ATdamtp.cam.ac.uk

Published by the American Physical Society under the terms of the Creative Commons Attribution 4.0 International license. Further distribution of this work must maintain attribution to the author(s) and the published article's title, journal citation, and DOI. Funded by SCOAP . understand this problem along two directions. First, the black hole entropies of static dyonic supersymmetric (BPS) black holes have been reproduced by topologically twisted indices of the $3 \mathrm{~d} \mathcal{N}=6$ superconformal theory $[4,5]$ by using supersymmetry localization [6]. Then there appeared agreement in various setups involving static magnetic charged black holes [7].

The second type of progress has been made in the canonical AdS/CFT correspondence between the $4 \mathrm{~d}$ $S U(N) \mathcal{N}=4$ super Yang-Mills theory (SYM) and type IIB superstring theory on $\mathrm{AdS}_{5} \times S^{5}$, which is also the subject of this paper. It is known that there are rotating electrically charged black hole solutions in $\mathrm{AdS}_{5}$ [8] which are embedded in the type IIB supergravity in $\mathrm{AdS}_{5} \times S^{5}$ as supersymmetric solutions with two supercharges $(1 / 16$ BPS $)^{1}[10]$. The black holes have three charges $\left(Q_{1}, Q_{2}\right.$, and $Q_{3}$ ) associated with $U(1)^{3} \subset S O(6)$ and two angular momenta $\left(J_{1}\right.$ and $\left.J_{2}\right)$ associated with the Cartan part of $S U(2)^{2} \sim S O(4) \subset S O(4,2)$. They are related to the black hole mass $M$ by

$$
M=g\left(\left|J_{1}\right|+\left|J_{2}\right|+\left|Q_{1}\right|+\left|Q_{2}\right|+\left|Q_{3}\right|\right),
$$

where $g$ is the gauge coupling. The Bekenstein-Hawking entropy of the black hole is [11]

\footnotetext{
${ }^{1}$ See also Ref. [9] for another embedding.
} 


$$
\begin{aligned}
S_{\mathrm{BH}} & =\frac{\text { area }}{4 G_{N}} \\
& =2 \pi \sqrt{Q_{1} Q_{2}+Q_{1} Q_{3}+Q_{2} Q_{3}-\frac{\pi}{4 G_{N} g^{3}}\left(J_{1}+J_{2}\right)},
\end{aligned}
$$

where the AdS/CFT dictionary between $G_{N} g^{3}$ and $N$ is

$$
\frac{\pi}{2 G_{N} g^{3}}=N^{2}
$$

A long-standing question is whether this black hole entropy is holographically explained by counting supersymmetric states preserving two supercharges (1/16-BPS states) in the $\mathcal{N}=4 \mathrm{SYM}$ on $S^{1} \times S^{3}$. Technically, it is much easier to analyze a supersymmetric index called the superconformal index $[12,13]$ rather than the net sum of the 1/16-BPS states:

$$
\begin{aligned}
I_{S^{1} \times S^{3}} & =\operatorname{Tr}\left[(-1)^{F} e^{-\beta\left\{Q, Q^{\dagger}\right\}} p^{J_{1}+(r / 2)} q^{J_{2}+(r / 2)} v_{1}^{q_{1}} v_{2}^{q_{2}}\right] \\
& =\operatorname{Tr}_{\mathrm{BPS}}\left[(-1)^{F} p^{J_{1}+(r / 2)} q^{J_{2}+(r / 2)} v_{1}^{q_{1}} v_{2}^{q_{2}}\right],
\end{aligned}
$$

where $r=\frac{2}{3}\left(Q_{1}+Q_{2}+Q_{3}\right)$ and $q_{1,2}=Q_{1,2}-Q_{3}$ taking charges of $U(1)^{3} \subset S O(6)_{R}$ symmetry to be $Q_{1,2,3} / 2$. One common worry is that the index may have a huge cancellation between bosonic and fermionic states due to the $(-1)^{F}$ with the fermion number $F$ so that it does not capture the black hole entropy [12] (see also Ref. [14] for other early attempts).

However, very recent papers have updated our understanding. First, Ref. [15] has shown that a Legendre transformation of the black hole entropy called the entropy function is given by a generalization of supersymmetric Casimir energy $E_{\text {Casimir }}[16,17]$ in the large- $N$ limit ${ }^{2}$ which is defined as a relative factor between the partition function and index ${ }^{3}$ :

$$
Z_{S^{1} \times S^{3}}=e^{-\beta E_{\text {Casimir }} I_{S^{1} \times S^{3}} .}
$$

Second, the authors of Ref. [20] have analyzed the index of the $U(N) \mathcal{N}=4 \mathrm{SYM}$ in a limit of shrinking $S^{1}$ at large $N$, which we refer to as the Cardy limit, and identified a saddle point of the holonomy integral which gives the black hole entropy function. Then they have assumed the dominance of the saddle point and derived the asymptotic behavior of the index in the Cardy limit which agrees with the black

\footnotetext{
${ }^{2}$ This claim had been made in an earlier version of Ref. [15] in arXiv. See a revised version for the update.

${ }^{3}$ The entropy function of the black hole was first computed in Ref. [18]. It was also argued in Ref. [18] that the entropy function is formally equal to the SUSY Casimir energy. The SUSY Casimir energy of the $\mathcal{N}=4$ SYM with the fugacities of $S O(6)_{R}$ was first computed in Ref. [19].
}

hole entropy (1.2) via a Legendre transformation with respect to the chemical potentials. They have also discussed a deconfinement transition in another paper [21]. Third, the authors of Ref. [22] have analyzed the index for $p=q$ in the large- $N$ limit by using a Bethe ansatz type formula of the index [23]. They have identified a saddle point which reproduces the black hole entropy function corresponding to the equal angular momenta case: $J_{1}=J_{2}$. They have also assumed that the saddle point is most dominant. It has also been stressed in Refs. [20-22] that the index with real fugacities has more cancellations than generic complex fugacities.

The aims of this paper are to provide further evidence that the index gives a microscopic explanation of the black hole entropy and make new predictions for the black hole physics in a more general case. We mainly study the supersymmetric index of the $S U(N) \mathcal{N}=4$ SYM on $S^{1} \times M_{3}$. We compute an asymptotic behavior of the index in the limit of shrinking $S^{1}$ for arbitrary $N$ by using a refinement $[24,25]$ of the supersymmetric Cardy formula [26], which is supposed to capture a large-charge limit of the black hole entropy. Therefore, our approach for the superconformal index case $\left(M_{3}=S^{3}\right)$ is basically the same as the one in Ref. [20]. The asymptotic behavior of the superconformal index at large $N$ agrees with the BekensteinHawking entropy (1.2) via a Legendre transformation with respect to the chemical potentials as already found in Ref. [20]. We also find that the agreement formally persists for finite $N$ if we slightly modify the AdS/CFT dictionary (1.3) as

$$
\left.\frac{\pi}{2 G_{N} g^{3}}\right|_{\text {finite } N}=N^{2}-1=4 c,
$$

where $c=\left(N^{2}-1\right) / 4$ is the central charge of the $S U(N)$ $\mathcal{N}=4$ SYM. This implies the existence of a nonrenormalization property for the black hole entropy function in the small- $S^{1}$ limit against quantum corrections. We also study the cases with other gauge groups and additional matter in conjugate representations and orbifold $\mathcal{N}=4 \mathrm{SYM}$. It turns out that the entropies of all the CFT examples in this paper are universally given by

$S_{\mathrm{QFT}}(Q, J)=2 \pi \sqrt{Q_{1} Q_{2}+Q_{1} Q_{3}+Q_{2} Q_{3}-2 c\left(J_{1}+J_{2}\right)}$,

with the central charge $c$. This formula is our prediction for the black hole entropy with full quantum corrections, which would give hints for what string theory is in a fully quantum mechanical sense. The results for other $M_{3}$ are also regarded as predictions to the gravity side. ${ }^{4}$ It is also interesting to note

\footnotetext{
${ }^{4}$ A proposal for quantum black hole entropy for the $M_{3}=$ $S^{1} \times T^{2}$ case has been made in Eq. (1.82) of Ref. [27].
} 
that the authors in Ref. [11] first wrote down the black hole formula for the dual of the $S U(N) \mathcal{N}=4 \mathrm{SYM}$ as

$S_{\mathrm{BH}}=2 \pi \sqrt{Q_{1} Q_{2}+Q_{1} Q_{3}+Q_{2} Q_{3}-2 c\left(J_{1}+J_{2}\right)}$

and then substituted $c=N^{2} / 4$ to get the formula

$S_{\mathrm{BH}}=2 \pi \sqrt{Q_{1} Q_{2}+Q_{1} Q_{3}+Q_{2} Q_{3}-\frac{N^{2}}{2}\left(J_{1}+J_{2}\right)}$

in their derivation. Of course, there is no difference in the large- $N$ limit, but our result suggests that Eq. (1.8) is more accurate for finite $N$.

Our argument for the $M_{3}=S^{3}$ case is overlapped with the one made in Ref. [20]. While the approach is the same up to technical details and the final result at large $N$ has been already obtained in Ref. [20], there are mainly three differences. First, we mainly consider the $S U(N) \mathcal{N}=$ 4 SYM rather than the $U(N)$ case, while the difference is irrelevant at large $N$, and we also finally consider the $\mathcal{N}=$ 4 SYM with a general gauge group as well as other theories. Second, we analyze the index for finite $N$, but we will see that the result in Ref. [20] is formally correct also for finite $N$. Finally, we not only identify a saddle point giving the black hole entropy (1.2) but also prove that the saddle point is most dominant. This amounts to justifying the assumption made in Ref. [20] at large $N$ and making sure that the most dominant contribution of the index gives the black hole entropy. Some contents discussed in Ref. [20] but not in this paper are the Macdonald limit [28] and the case for $\mathrm{AdS}_{7}$ black holes.

\section{ASYMPTOTIC BEHAVIOR OF THE SUPERSYMMETRIC INDEX IN $S U(N) \mathcal{N}=4$ SYM}

Let us consider the $S U(N) \mathcal{N}=4 \mathrm{SYM}$ on $S_{\beta}^{1} \times M_{3}$ with the radius $\beta$. We take $M_{3}$ to preserve a part of supersymmetry, and this constrains $S_{\beta}^{1} \times M_{3}$ to be complex [29]. Different choices of $M_{3}$ count different quantum numbers, as different $M_{3}$ 's have different isometries. One of the most well-studied cases is the index on $S^{1} \times S^{3}$ known as the superconformal index $[12,13]$ :

$$
I_{S^{1} \times S^{3}}=\operatorname{Tr}_{\mathrm{BPS}}\left[(-1)^{F} p^{J_{1}+(r / 2)} q^{J_{2}+(r / 2)} v_{1}^{q_{1}} v_{2}^{q_{2}}\right],
$$

where

$$
p=e^{2 \pi i \sigma}, \quad q=e^{2 \pi i \tau}, \quad v_{1,2}=e^{2 \pi i m_{1,2}} .
$$

We are interested in an asymptotic behavior of the partition function in the shrinking $S^{1}$ limit: $\beta \rightarrow 0$. In this limit, the partition function is exactly the same as the supersymmetric index, since we can ignore the contribution from the SUSY Casimir energy in Eq. (1.5). Therefore, we are essentially looking at the asymptotic behavior of the index. There is a general formula to describe such asymptotic behavior for the general $4 \mathrm{~d} \mathcal{N}=1$ SUSY theory with $U(1)_{R}$ symmetry and a Lagrangian description which is a refinement [24,25] of the SUSY Cardy formula [26].

For simplicity of explanation, we first consider the superconformal index, which is defined through the supersymmetric partition function on a space with a topology of $S^{1} \times S^{3}$. For example, if we take $M_{3}$ to be the squashed sphere $S_{b}^{3}, \tau$ and $\sigma$ are given by $\tau=-\beta b / 2 \pi i$ and $\sigma=-\beta b^{-1} / 2 \pi i$, respectively. For any choices, the Cardy limit $\beta \rightarrow 0$ for the superconformal index is equivalent to $|\tau|,|\sigma| \rightarrow 0$. The refined SUSY Cardy formula for the superconformal index is given by ${ }^{5}$

$$
\begin{aligned}
I_{S^{1} \times S^{3}} \underset{|\tau|,|\bar{\sigma}| \rightarrow 0}{\simeq} e^{-[i \pi(\tau+\sigma) / 12 \tau \sigma] \operatorname{Tr}(R)} \\
\quad \times \int d^{\mathrm{rankG}} a e^{i \pi / 6 \tau \sigma V_{2}(a)+[i \pi(\tau+\sigma) / 2 \tau \sigma] V_{1}(a)},
\end{aligned}
$$

which has been derived in two ways: taking the limit in the localization formula [25] and effective theory consideration [24].

Several definitions are in order. First, $G$ is the gauge group, and $e^{2 \pi i a_{j}}$ with $j=1, \ldots, \operatorname{rank} G$ is holonomy around $S^{1}$ valued in the maximal torus of $G$. Second, $\operatorname{Tr}(R)$ is the anomaly coefficient ${ }^{6}$ of the $U(1)_{R}$ symmetry and related to conformal anomalies by $\operatorname{Tr}(R)=-16(c-a)$ for the superconformal case. Third, $V_{2}(a)$ and $V_{1}(a)$ are piecewise polynomials of $a_{j}$ and flavor chemical potentials whose forms are explicitly determined if we specify representations, $U(1)_{R}$ charges, and flavor charges of chiral multiplets (see the Appendix). Their explicit forms for the $S U(N)$ $\mathcal{N}=4$ SYM are $^{7}$

$$
\begin{aligned}
V_{2}(a)= & -\sum_{1 \leq i \neq j \leq N}\left[\kappa\left(a_{i j}+m_{1}\right)+\kappa\left(a_{i j}+m_{2}\right)\right. \\
& \left.+\kappa\left(a_{i j}-m_{1}-m_{2}\right)\right] \\
& -(N-1)\left[\kappa\left(m_{1}\right)+\kappa\left(m_{2}\right)+\kappa\left(-m_{1}-m_{2}\right)\right], \\
V_{1}(a)= & \frac{1}{3} \sum_{1 \leq i \neq j \leq N}\left[3 \theta\left(a_{i j}\right)-\theta\left(a_{i j}+m_{1}\right)-\theta\left(a_{i j}+m_{2}\right)\right. \\
& \left.-\theta\left(a_{i j}-m_{1}-m_{2}\right)\right] \\
& -\frac{N-1}{3}\left[\theta\left(m_{1}\right)+\theta\left(m_{2}\right)+\theta\left(-m_{1}-m_{2}\right)\right],
\end{aligned}
$$

where

\footnotetext{
${ }^{5}$ See Ref. [30] for earlier related works.

${ }^{6}$ This is simply the sum of $U(1)_{R}$ charges of fermions in the theory under consideration.

${ }^{7}$ For $m_{1}=0=m_{2}, V_{2}(a)$ and $V_{1}(a)$ are zero. The leading asymptotic behavior of the index for this case is $(N-1) \log \beta$ as shown in Ref. [25].
} 


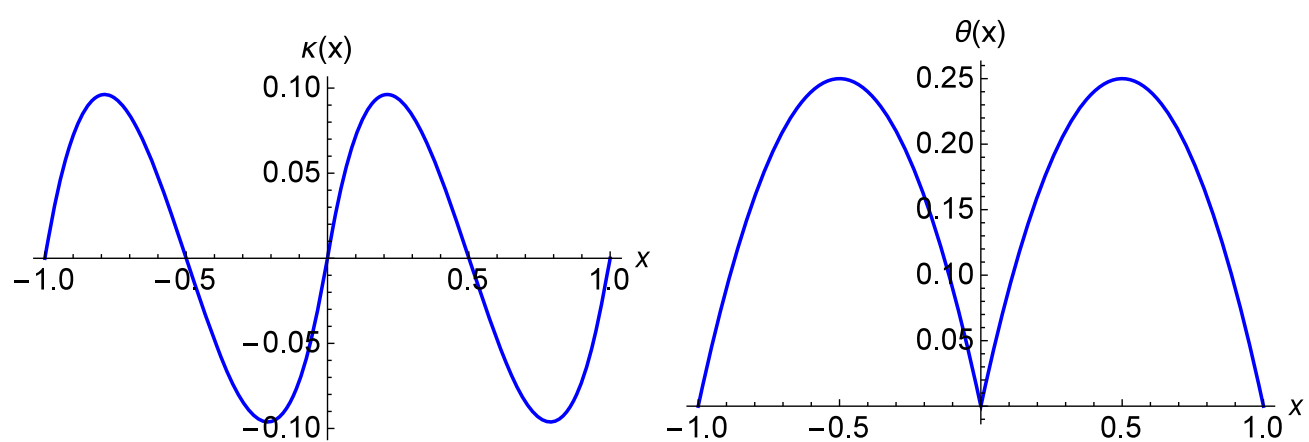

FIG. 1. $\kappa(x)$ and $\theta(x)$.

$$
a_{i j}=a_{i}-a_{j}, \quad \sum_{j=1}^{N} a_{j}=0
$$

$\kappa(x)=\{x\}(1-\{x\})(1-2\{x\}), \quad \theta(x)=\{x\}(1-\{x\})$,

with fractional part $\{x\} \equiv x-[x]$ (see Fig. 1). $V_{2}(a)$ $\left[V_{1}(a)\right]$ is apparently a piecewise cubic (quadratic) polynomial, but this is actually quadratic (linear) because of anomaly cancellations involving the gauge symmetry.

Here we restrict ourselves to

$$
\operatorname{Re}\left(\frac{i}{\tau \sigma}\right)<0
$$

and $\operatorname{Im}\left(m_{1,2}\right)=\mathcal{O}(\beta)$. We will mention other regimes later. In this regime, the integral in the limit is dominated by the saddle point configuration(s) to minimize the function $V_{2}(a)$. We can easily find a dominant saddle point as follows. Noting $\kappa(-x)=-\kappa(x)$ and $^{8} \kappa(x+1)=\kappa(x)$, we rewrite $V_{2}(a)$ as

$$
V_{2}(a)=\sum_{i<j} f\left(a_{i j}\right)+\frac{N-1}{2} f(0),
$$

where

$$
\begin{aligned}
f\left(a_{i j}\right)= & \kappa\left(a_{i j}-\left\{m_{1}\right\}\right)-\kappa\left(a_{i j}+\left\{m_{1}\right\}\right)+\kappa\left(a_{i j}-\left\{m_{2}\right\}\right) \\
& -\kappa\left(a_{i j}+\left\{m_{2}\right\}\right)+\kappa\left(a_{i j}+\left\{m_{1}\right\}+\left\{m_{2}\right\}\right) \\
& -\kappa\left(a_{i j}-\left\{m_{1}\right\}-\left\{m_{2}\right\}\right) .
\end{aligned}
$$

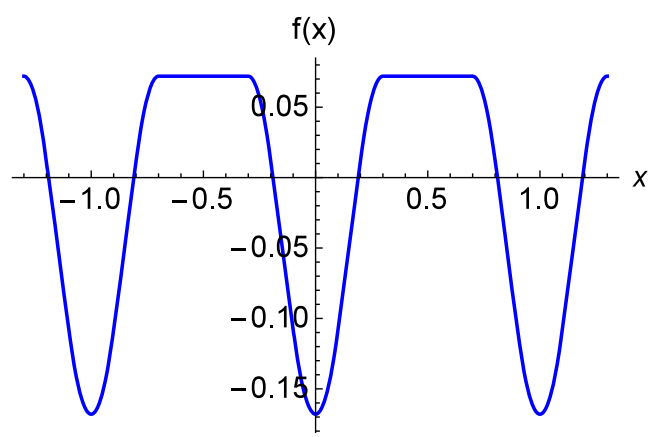

FIG. 2. $f(x)$ for $\left(m_{1}, m_{2}\right)=(0.2,0.1)$.

It is sufficient to minimize each $f\left(a_{i j}\right)$ and show that we can realize a simultaneously minimizing configuration. As a result, the minimizing configuration is simply $a_{j}=0$ for any $j$ as illustrated in Fig. 2 for a specific value of $\left(m_{1}, m_{2}\right)$. To see this generally, it is convenient to first analyze the regime

$$
0 \leq\left\{m_{2}\right\} \leq\left\{m_{1}\right\}, \quad\left\{m_{1}\right\}+\left\{m_{2}\right\} \leq \frac{1}{2}
$$

and extend it to other regimes by using the periodicity $m_{1,2} \sim m_{1,2}+1$. In this regime, noting $\kappa(x)=2 x^{3}-$ $3 x|x|+x$ for $|x| \leq 1$, the function $f(x)$ in "the fundamental region" $|x|<1-\left\{m_{1}\right\}+\left\{m_{2}\right\}$ is given by

$$
f(x)= \begin{cases}6 x^{2}+12\left\{m_{1}\right\}\left\{m_{2}\right\}\left(\left\{m_{1}\right\}+\left\{m_{2}\right\}-1\right) & \text { for }|x| \leq\left\{m_{2}\right\}, \\ 12 m_{2}|x|+6 m_{2}\left(2 m_{1}^{2}+2 m_{1} m_{2}-2 m_{1}-m_{2}\right) & \text { for }\left\{m_{2}\right\} \leq|x| \leq\left\{m_{1}\right\}, \\ -6\left(|x|-\left\{m_{1}\right\}-\left\{m_{2}\right\}\right)^{2}+12\left\{m_{1}\right\}\left\{m_{2}\right\}\left(\left\{m_{1}\right\}+\left\{m_{2}\right\}\right) & \text { for }\left\{m_{1}\right\} \leq|x| \leq\left\{m_{1}\right\}+\left\{m_{2}\right\}, \\ 12\left\{m_{1}\right\}\left\{m_{2}\right\}\left(\left\{m_{1}\right\}+\left\{m_{2}\right\}\right) & \text { for }\left\{m_{1}\right\}+\left\{m_{2}\right\} \leq|x|,\end{cases}
$$

\footnotetext{
${ }^{8}$ Physically, this periodicity reflects invariance under a large gauge transformation.

$\left.f(x)\right|_{\min }=f(0)=12\left\{m_{1}\right\}\left\{m_{2}\right\}\left(\left\{m_{1}\right\}+\left\{m_{2}\right\}-1\right)$.
} 
Therefore, the minimum of $V_{2}(a)$ is realized by $a_{i j}=0$ for all $i, j$ with the traceless condition $\sum_{j=1}^{N} a_{j}=0$, which is nothing but $a_{j}=0$. Thus, we find the minimum of $V_{2}(a)$ as

$$
\begin{aligned}
\left.V_{2}(a)\right|_{\min } & =V_{2}(0) \\
& =6\left(N^{2}-1\right)\left\{m_{1}\right\}\left\{m_{2}\right\}\left(\left\{m_{1}\right\}+\left\{m_{2}\right\}-1\right) .
\end{aligned}
$$

The next order $\mathcal{O}\left(\beta^{-1}\right)$ is simply obtained by substituting ${ }^{9}$ the saddle point into $V_{1}(a)$ :

$$
\begin{aligned}
\left.V_{1}(a)\right|_{a_{j}=0}= & \frac{2\left(N^{2}-1\right)}{3}\left[\left\{m_{1}\right\}^{2}+\left\{m_{2}\right\}^{2}\right. \\
& \left.+\left\{m_{1}\right\}\left\{m_{2}\right\}-\left\{m_{1}\right\}-\left\{m_{2}\right\}\right] .
\end{aligned}
$$

Then, noting $c-a=0$ in the $\mathcal{N}=4 \mathrm{SYM}$, we find the Cardy limit of the superconformal index to be

$$
\begin{aligned}
\log I_{S^{1} \times S^{3}} \underset{|\tau|,|\overline{\mid}| \rightarrow 0}{\simeq} \frac{i \pi\left(N^{2}-1\right)}{\tau \sigma}\left[\left\{m_{1}\right\}\left\{m_{2}\right\}\left(\left\{m_{1}\right\}+\left\{m_{2}\right\}-1\right)\right. \\
\quad+\frac{\tau+\sigma}{3}\left(\left\{m_{1}\right\}^{2}+\left\{m_{2}\right\}^{2}+\left\{m_{1}\right\}\left\{m_{2}\right\}\right. \\
\left.\left.\quad-\left\{m_{1}\right\}-\left\{m_{2}\right\}\right)\right] .
\end{aligned}
$$

In order to directly compare this with the BekensteinHawking entropy, it is convenient to rewrite the result in the following two steps. First, we redefine the chemical potentials $m_{1,2}$ as

$$
m_{1,2}=\Delta_{1,2}-\frac{\tau+\sigma}{3},
$$

so that our index becomes

$$
\operatorname{Tr}_{\mathrm{BPS}}\left[(-1)^{F} p^{J_{1}+Q_{3}} q^{J_{2}+Q_{3}} e^{2 \pi i \Delta_{1}\left(Q_{1}-Q_{3}\right)} e^{2 \pi i \Delta_{2}\left(Q_{2}-Q_{3}\right)}\right] .
$$

This object is the same as the grand canonical partition function

$$
\operatorname{Tr}_{\mathrm{BPS}}\left[p^{J_{1}} q^{J_{2}} \prod_{a=1}^{3} e^{2 \pi i \Delta_{a} Q_{a}}\right],
$$

with the constraint ${ }^{10} \Delta_{1}+\Delta_{2}+\Delta_{3}-\tau-\sigma-1 \in 2 \mathbb{Z}$. In this parametrization, the asymptotic behavior of the index becomes

\footnotetext{
${ }^{9}$ The saddle point of $V_{2}(a)$ also realizes the minimum of $V_{1}(a)$ as a result, though this property is not necessary for our analysis.

${ }^{10}$ Note that $(-1)^{F}=e^{2 \pi i Q_{3}}$.
}

$$
\begin{aligned}
& \log I_{S^{1} \times S^{3}} \underset{|\tau|,|\sigma| \rightarrow 0}{\simeq} \\
& \frac{i \pi\left(N^{2}-1\right)\left\{\Delta_{1}\right\}\left\{\Delta_{2}\right\}\left(\left\{\Delta_{1}\right\}+\left\{\Delta_{2}\right\}-1-\sigma-\tau\right)}{\tau \sigma} .
\end{aligned}
$$

Second, we make a Legendre transformation [18] with respect to $\left(\sigma, \tau, \Delta_{1}, \Delta_{2}\right)$ to directly obtain entropy or equivalently degeneracy of states with fixed charges and angular momenta as explained in the next subsection.

\section{A. Comments on other regimes of $(\boldsymbol{\tau}, \boldsymbol{\sigma})$}

If we take another regime $\operatorname{Re}\left(\frac{i}{\tau \sigma}\right)>0$, then we need to minimize $-V_{2}(a)$ or, equivalently, maximize $V_{2}(a)$. Then the dominant saddle points are given by the points maximizing $f(x)$. According to Eq. (2.10), the saddle points are any configurations giving the plateau regime of $f(x)$, namely, the ones satisfying $\left\{m_{1}\right\}+\left\{m_{2}\right\} \leq\left|\left\{a_{i j}\right\}\right|<$ $1-\left\{m_{1}\right\}+\left\{m_{2}\right\}$. We immediately see that the saddle points are no longer isolated, and, therefore, it remains integration over the saddle points which seems complicated, since $V_{1}(a)$ is not constant in this regime. As a result, the asymptotic behavior of the index is

$$
\begin{aligned}
\log I_{S^{1} \times S^{3}} \underset{|\tau|,|\bar{\sigma}| \rightarrow 0}{\simeq} \frac{i \pi\left\{m_{1}\right\}\left\{m_{2}\right\}}{\tau \sigma} \\
\times\left[\left(N^{2}-1\right)\left(\left\{m_{1}\right\}+\left\{m_{2}\right\}\right)-(N-1)\right] \\
+\log \int_{\text {saddles }} d^{N} a \delta\left(\sum_{j=1}^{N} a_{j}\right) e^{i \pi(\tau+\sigma) / 2 \tau \sigma V_{1}(a)} .
\end{aligned}
$$

This implies that we have an anti-Stokes line at $\operatorname{Re}\left(\frac{i}{\tau \sigma}\right)=0$, since the dominant saddle point changes there. The above saddle points are unstable in the regime $\operatorname{Re}\left(\frac{i}{\tau \sigma}\right)<0$, which we have mainly considered in this paper. Relatedly, Stokes phenomena have been observed in the large- $N$ analysis of the Bethe ansatz type formula [22]. It would be interesting to understand the above phenomena in more detail and find their physical interpretations especially from the gravity side. This might be related to hairy black holes [31].

\section{B. Comparison with Bekenstein-Hawking entropy}

This subsection is essentially a review of various papers $[15,18,20,22]$ up to identifications of parameters and final results. ${ }^{11}$ The Legendre transformation of the black hole entropy is referred to as the entropy function [33]. Suppose that we have the entropy function $\mathcal{S}$ :

\footnotetext{
${ }^{11}$ The original argument was in Sec. 3 of Ref. [18]. This subsection is also a review of Appendix B of Ref. [15], Sec. 2.3 of Ref. [20], and Sec. 6 of Ref. [22]. See also Ref. [32] for a similar argument for $\mathrm{AdS}_{7}$ black holes.
} 


$$
\mathcal{S}=2 \pi i \nu \frac{X_{1} X_{2} X_{3}}{\omega_{1} \omega_{2}},
$$

with the constraint

$$
X_{1}+X_{2}+X_{3}-\omega_{1}-\omega_{2}=n .
$$

These quantities in our case are

$$
\begin{aligned}
\mathcal{S} & =-\log I_{S^{1} \times S_{b}^{3}}, \quad \nu=\frac{N^{2}-1}{2}, \\
\omega_{1}=\sigma, \quad \omega_{2}=\tau, & X_{a}=\left\{\Delta_{a}\right\}, \quad n=1 .
\end{aligned}
$$

The entropy $S(Q, J)$ is obtained by the Legendre transformation

$$
\begin{aligned}
S(Q, J)= & \mathcal{S}\left(X_{a}, \omega_{i}\right)+2 \pi i\left(\sum_{a=1}^{3} X_{a} Q_{a}+\sum_{I=1}^{2} \omega_{I} J_{I}\right) \\
& +\left.2 \pi i \Lambda\left(\sum_{a=1}^{3} X_{a}-\sum_{I=1}^{2} \omega_{I}-n\right)\right|_{X_{a}, \omega_{i}},
\end{aligned}
$$

where $\Lambda$ is the Lagrange multiplier. The extremization conditions are

$$
\frac{\partial \mathcal{S}}{\partial X_{a}}=-2 \pi i\left(Q_{a}+\Lambda\right), \quad \frac{\partial \mathcal{S}}{\partial \omega_{I}}=-2 \pi i\left(J_{I}-\Lambda\right),
$$

with the constraint (2.21). Note that we do not need explicit solutions for $\left(X_{a}, \omega_{I}\right)$ to compute $S$ if we use the relation

$$
\mathcal{S}=\sum_{a=1}^{3} X_{a} \frac{\partial \mathcal{S}}{\partial X_{a}}+\sum_{I=1}^{2} \omega_{I} \frac{\partial \mathcal{S}}{\partial \omega_{I}}
$$

Then the entropy is simply given by

$$
S=2 \pi i n \Lambda,
$$

where $\Lambda$ satisfies

$$
\begin{aligned}
0 & =\left(Q_{1}+\Lambda\right)\left(Q_{2}+\Lambda\right)\left(Q_{3}+\Lambda\right)+\nu\left(J_{1}-\Lambda\right)\left(J_{2}-\Lambda\right) \\
& =\Lambda^{3}+p_{2} \Lambda^{2}+p_{1} \Lambda+p_{0},
\end{aligned}
$$

with

$$
\begin{aligned}
& p_{0}=Q_{1} Q_{2} Q_{3}+\nu J_{1} J_{2}, \\
& p_{1}=Q_{1} Q_{2}+Q_{2} Q_{3}+Q_{3} Q_{1}-\nu\left(J_{1}+J_{2}\right), \\
& p_{2}=Q_{1}+Q_{2}+Q_{3}+\nu J_{1} J_{2} .
\end{aligned}
$$

The equation for $\Lambda$ has the three solutions $\Lambda=\left\{-p_{2}, \pm i \sqrt{p_{1}}\right\}$ with $p_{1}, p_{2} \in \mathbb{R}_{\geq 0}$. Imposing $S \in \mathbb{R}_{\geq 0}$, the physical solution among the three is $\Lambda=-i \operatorname{sgn}(n) \sqrt{p_{1}}$. which leads us to the entropy

$$
S=2 \pi|n| \sqrt{p_{1}} .
$$

Under the identifications (2.22), the entropy computed by the superconformal index of the $S U(N) \mathcal{N}=4 \mathrm{SYM}$ is

$S_{\mathrm{QFT}}(Q, J)$

$$
=2 \pi \sqrt{Q_{1} Q_{2}+Q_{1} Q_{3}+Q_{2} Q_{3}-\frac{N^{2}-1}{2}\left(J_{1}+J_{2}\right)},
$$

which agrees with the Bekenstein-Hawking entropy (1.2) via the AdS/CFT dictionary (1.3) in the large- $N$ limit. Interestingly, the agreement persists for finite $N$ if we slightly modify the AdS/CFT dictionary for finite $N$ as

$$
\left.\frac{\pi}{2 G_{N} g^{3}}\right|_{\text {finite } N}=N^{2}-1=4 c,
$$

where $c=\frac{N^{2}-1}{4}$ is the central charge. This may suggest that the black hole entropy with full quantum corrections is captured by the Bekenstein-Hawking entropy with the renormalized Newton constant (2.31) in the Cardy limit.

\section{General $M_{3}$}

The refined SUSY Cardy formula for the $S U(N) \mathcal{N}=4$ $\mathrm{SYM}$ on $S^{1} \times M_{3}$ is

$$
I_{S^{1} \times M_{3}} \underset{\beta \rightarrow 0}{\simeq} \int d^{N} a \delta\left(\sum_{j=1}^{N} a_{j}\right) e^{-\left(\pi^{3} i A_{M_{3}} / 6 \beta^{2}\right) V_{2}(a)+\left(\pi^{2} L_{M_{3}} / 2 \beta\right) V_{1}(a)-(1 / 2 \beta) \tilde{V}_{1}(a)},
$$

where $\tilde{V}_{1}(a)$ is the contribution absent in the superconformal index:

$$
\tilde{V}_{1}(a)=\sum_{i \neq j}\left(\ell_{M_{3}}^{i}-\ell_{M_{3}}^{j}\right)\left[\theta\left(a_{i j}+m_{1}\right)+\theta\left(a_{i j}+m_{2}\right)+\theta\left(a_{i j}-m_{1}-m_{2}\right)+\theta\left(a_{i j}\right)\right] .
$$


The quantities $A_{M_{3}}, L_{M_{3}}$, and $\ell_{M_{3}}^{i}$ are local functionals on $M_{3}$ given by bosonic fields in the $3 \mathrm{~d}$ new minimal supergravity multiplet $\left(h_{\mu \nu}, A_{\mu}^{(R)}, H, c_{\mu}\right)$ and $3 \mathrm{~d} \mathcal{N}=2$ vector multiplet $^{12}\left(A_{\mu}, \sigma, D\right)$ :

$$
\begin{aligned}
A_{M_{3}} & =\frac{i}{\pi^{2}} \int_{M_{3}} d^{3} x \sqrt{h}\left[-c^{\mu} v_{\mu}+2 H\right], \\
L_{M_{3}} & =\frac{1}{\pi^{2}} \int_{M_{3}} d^{3} x \sqrt{h}\left[-A_{\mu}^{(R) \mu} v^{\mu}+v_{\mu} v^{\mu}-\frac{1}{2} H^{2}+\frac{1}{4} R\right], \\
\ell_{M_{3}}^{i} & =\frac{1}{\pi^{2}} \int_{M_{3}} d^{3} x \sqrt{h}\left[-A_{\mu}^{i} v^{\mu}+D^{i}\right],
\end{aligned}
$$

which come from induced Chern-Simons terms of $U(1)_{\mathrm{KK}}-U(1)_{\mathrm{KK}}, U(1)_{\mathrm{KK}}-U(1)_{R}$, and $U(1)_{\mathrm{KK}}$-gauge or flavor, respectively, from the viewpoint of the $3 \mathrm{~d}$ effective theory ${ }^{13}$ on $M_{3}$. Technically, $A_{M_{3}}$ and $L_{M_{3}}$ are just constants for fixed $M_{3}$, while $l_{M_{3}}^{i}$ generally depends on (supersymmetric configurations of) the dynamical vector multiplets, though it has typically a simple form because of SUSY. ${ }^{14}$

Here we restrict ourselves to

$$
\operatorname{Re}\left(\frac{i A_{M_{3}}}{\beta^{2}}\right)>0
$$

which generalizes the condition (2.6). Then the integral in the $\beta \rightarrow 0$ limit is dominated by the saddle point of $V_{2}(a)$ which is already found as $a_{j}=0$. Thus, noting $\left.\tilde{V}_{1}(a)\right|_{a_{j}=0}=0$, the asymptotic behavior of the index for general $M_{3}$ is

$$
\begin{aligned}
\log I_{S^{1} \times M_{3}} \underset{\beta \rightarrow 0}{\simeq} & -\frac{2 \pi^{3} i A_{M_{3}}\left(N^{2}-1\right)}{\beta^{2}}\left\{m_{1}\right\}\left\{m_{2}\right\}\left(\left\{m_{1}\right\}\right. \\
& \left.+\left\{m_{2}\right\}-1\right)+\frac{\pi^{2} L_{M_{3}}\left(N^{2}-1\right)}{3 \beta} \\
& \times\left[\left\{m_{1}\right\}^{2}+\left\{m_{2}\right\}^{2}+\left\{m_{1}\right\}\left\{m_{2}\right\}-\left\{m_{1}\right\}-\left\{m_{2}\right\}\right] .
\end{aligned}
$$

This makes predictions to the gravity side for more general $M_{3}$. For example, the case for a lens space index is

$$
\log I_{S^{1} \times S^{3} / \mathbb{Z}_{n}} \underset{|\tau|,|\bar{\sigma}| \rightarrow 0}{\simeq} \frac{\log I_{S^{1} \times S^{3}}}{n} .
$$

\footnotetext{
${ }^{12}$ This is for both gauge and global symmetries.

${ }^{13}$ See Refs. [24,26] for details.

${ }^{14}$ For example, $\ell_{S_{3} / \mathbb{Z}_{n}}^{i}=0$ and $\ell_{S^{1} \times \Sigma_{g}}^{i} \propto$ (magnetic charge) with Riemann surface $\Sigma_{g}^{n}$.
}

\section{GENERALIZATIONS}

\section{A. Other gauge groups}

Generalization to other gauge groups is straightforward, because we can still apply the technique in the $S U(N)$ case. For the $\mathcal{N}=4$ SYM with gauge group $G$, the functions appearing in the SUSY Cardy formula are

$$
\begin{aligned}
V_{2}(a)= & -\sum_{\alpha \in \operatorname{root}}\left[\kappa\left(\alpha \cdot a+m_{1}\right)+\kappa\left(\alpha \cdot a+m_{2}\right)\right. \\
& \left.+\kappa\left(\alpha \cdot a-m_{1}-m_{2}\right)\right] \\
& -\operatorname{rank}(G)\left[\kappa\left(m_{1}\right)+\kappa\left(m_{2}\right)+\kappa\left(-m_{1}-m_{2}\right)\right], \\
V_{1}(a)= & \frac{1}{3} \sum_{\alpha \in \operatorname{root}}\left[3 \theta(\alpha \cdot a)-\theta\left(\alpha \cdot a+m_{1}\right)-\theta\left(\alpha \cdot a+m_{2}\right)\right. \\
& \left.-\theta\left(\alpha \cdot a-m_{1}-m_{2}\right)\right] \\
& -\frac{N-1}{3}\left[\theta\left(m_{1}\right)+\theta\left(m_{2}\right)+\theta\left(-m_{1}-m_{2}\right)\right], \\
\tilde{V}_{1}(a)= & \sum_{\alpha \in \operatorname{root}} \alpha \cdot \ell_{M_{3}}\left[\theta\left(\alpha \cdot a+m_{1}\right)+\theta\left(\alpha \cdot a+m_{2}\right)\right. \\
& \left.+\theta\left(\alpha \cdot a-m_{1}-m_{2}\right)+\theta(\alpha \cdot a)\right] .
\end{aligned}
$$

In terms of $f(x)$, we rewrite $V_{2}(a)$ as

$$
V_{2}(a)=\sum_{\alpha \in \text { root }_{+}} f(\alpha \cdot a)+\frac{\operatorname{rank}(G)}{2} f(0)
$$

which has the global minimum at $a_{j}=0$ by the same logic ${ }^{15}$ as in Sec. II. Thus, the index asymptotically behaves as

$$
\begin{aligned}
\log I_{S^{1} \times M_{3}} \underset{\beta \rightarrow 0}{\simeq}-\frac{2 \pi^{3} i A_{M_{3}} \operatorname{dim}(G)}{\beta^{2}}\left\{m_{1}\right\}\left\{m_{2}\right\}\left(\left\{m_{1}\right\}+\left\{m_{2}\right\}-1\right) \\
+\frac{\pi^{2} L_{M_{3}} \operatorname{dim}(G)}{3 \beta}\left[\left\{m_{1}\right\}^{2}+\left\{m_{2}\right\}^{2}\right. \\
\left.\quad+\left\{m_{1}\right\}\left\{m_{2}\right\}-\left\{m_{1}\right\}-\left\{m_{2}\right\}\right] .
\end{aligned}
$$

Especially, the superconformal index is ${ }^{16}$

$$
\begin{aligned}
& \log I_{S^{1} \times S^{3}} \\
& \underset{|\tau|,|\bar{\sigma}| \rightarrow 0}{\widetilde{\tau}} \frac{i \pi \operatorname{dim} G\left\{\Delta_{1}\right\}\left\{\Delta_{2}\right\}\left(\left\{\Delta_{1}\right\}+\left\{\Delta_{2}\right\}-1-\sigma-\tau\right)}{\tau \sigma} .
\end{aligned}
$$

\footnotetext{
${ }^{15}$ For $G=U(N)$, this is sufficient but not necessary due to decoupling the diagonal $U(1)$. The same minimum is realized by any configuration satisfying $a_{1}=\cdots=a_{N}$, which is the same as the one obtained in Ref. [20]. This flat direction affects $\mathcal{O}(\log \beta)$.

${ }^{16}$ For $G=U(N)$, the result is the same as the one obtained in Ref. [20], which takes the large- $N$ limit. However, our result shows that the result of Ref. [20] is formally correct also for finite $N$. This implies that contributions which are ignored in Ref. [20] vanish in the Cardy limit.
} 
The Legendre transformation leads us to the entropy

$$
\begin{aligned}
S_{\mathrm{QFT}}(Q, J) & =2 \pi \sqrt{Q_{1} Q_{2}+Q_{1} Q_{3}+Q_{2} Q_{3}-\frac{\operatorname{dim} G}{2}\left(J_{1}+J_{2}\right)} \\
& =2 \pi \sqrt{Q_{1} Q_{2}+Q_{1} Q_{3}+Q_{2} Q_{3}-2 c\left(J_{1}+J_{2}\right)},
\end{aligned}
$$

where we have used $c=\operatorname{dim} G / 4$. This implies that the dual black hole entropy for gauge group $G$ is captured by (1.2) under the identification

$$
\left.\frac{\pi}{2 G_{N} g^{3}}\right|_{\text {finiteN }}=4 c
$$

even if $G$ is not necessarily $S U(N)$ or $U(N)$.

\section{B. Adding matter in conjugate representations}

Let us add pairs of chiral multiplets in conjugate representations to the $\mathcal{N}=4 \mathrm{SYM}$ with the gauge group $G$. In general, this theory may have new flavor symmetries, but let us keep their fugacities turned off for simplicity. For this case, the function $V_{2}(a)$ does not receive contributions from the matter because of $\kappa(-x)=-\kappa(x)$. Therefore, the holonomy integral of the SUSY Cardy formula is still dominated by $a_{j}=0$. Furthermore, contributions from the additional matter to the $V_{1}(a)$ and $\tilde{V}_{1}(a)$ are zero at $a_{j}=0$. Thus, the asymptotic behavior of the index is

$$
\begin{aligned}
\log I_{S^{1} \times M_{3}} \underset{\beta \rightarrow 0}{\simeq} & -\frac{\pi^{2} L_{M_{3}}}{12 \beta} \operatorname{Tr}(R) \\
& -\frac{2 \pi^{3} i A_{M_{3}} \operatorname{dim}(G)}{\beta^{2}}\left\{m_{1}\right\}\left\{m_{2}\right\}\left(\left\{m_{1}\right\}+\left\{m_{2}\right\}-1\right) \\
& +\frac{\pi^{2} L_{M_{3}} \operatorname{dim}(G)}{3 \beta}\left[\left\{m_{1}\right\}^{2}+\left\{m_{2}\right\}^{2}\right. \\
& \left.+\left\{m_{1}\right\}\left\{m_{2}\right\}-\left\{m_{1}\right\}-\left\{m_{2}\right\}\right] .
\end{aligned}
$$

Note that only the difference from the $\mathcal{N}=4$ SYM is the first term, which is simply captured by the unrefined SUSY Cardy formula [26]. Specifying to the superconformal index case, we find

$$
\begin{aligned}
\log I_{S^{1} \times S^{3}} \underset{|\tau|,|\bar{\sigma}| \rightarrow 0}{\simeq} \frac{i \pi \operatorname{dim} G\left\{\Delta_{1}\right\}\left\{\Delta_{2}\right\}\left(\left\{\Delta_{1}\right\}+\left\{\Delta_{2}\right\}-1-\sigma-\tau\right)}{\tau \sigma} \\
\quad-\frac{i \pi(\tau+\sigma)}{12 \tau \sigma} \operatorname{Tr}(R) .
\end{aligned}
$$

This indicates that the entropies in theories with $|\operatorname{Tr}(R)| / N^{2} \ll 1$ in the large- $N$ limit are universally captured by the one of the $\mathcal{N}=4$ SYM.

\section{Orbifold $\mathcal{N}=4$ SYM}

Let us consider so-called orbifold $\mathcal{N}=4$ SYM which is the circular quiver $\mathcal{N}=2$ gauge theory with the $U(N)_{1} \times \cdots U(N)_{K}$ gauge group and one bifundamental hypermultiplet of neighboring gauge group $U(N)_{I} \times$ $U(N)_{I+1}$. We turn on chemical potentials $m_{1}$ and $m_{2}$ of flavor symmetry $U(1)_{1} \times U(1)_{2}$ in which the $U(1)_{1}$ $\left(U(1)_{2}\right)$ symmetry assigns charge 1 to each $\mathcal{N}=1$ (anti) bifundamental chiral multiplet and charge -1 to each $\mathcal{N}=1$ adjoint chiral multiplet in the $\mathcal{N}=2$ vector multiplet. The function $V_{2}(a)$ for this theory is

$$
\begin{aligned}
V_{2}(a)= & -\sum_{I=1}^{K} \sum_{1 \leq i, j \leq N}\left[\kappa\left(a_{i}^{(I)}-a_{j}^{(I+1)}+m_{1}\right)\right. \\
& \left.+\kappa\left(-a_{i}^{(I)}+a_{j}^{(I+1)}+m_{2}\right)+\kappa\left(a_{i j}^{(I)}-m_{1}-m_{2}\right)\right] .
\end{aligned}
$$

It is not easy to find the global minimum of this function in contrast to the $\mathcal{N}=4 \mathrm{SYM}$. Instead of solving this problem completely, we proceed by taking the physically motivated ansatz:

$$
a_{j}^{(I)}=a_{j}^{(J)}=a_{j},
$$

which reflects $\mathbb{Z}_{k}$ rotation symmetry of the quiver diagram or, equivalently, all the gauge groups are "democratic."17 Under this ansatz, $V_{2}(a)$ becomes

$$
\begin{aligned}
\left.V_{2}(a)\right|_{a_{j}^{(I)}=a_{j}^{(J)}=a_{j}}= & -K \sum_{1 \leq i, j \leq N}\left[\kappa\left(a_{i j}+m_{1}\right)+\kappa\left(-a_{i j}+m_{2}\right)\right. \\
& \left.+\kappa\left(a_{i j}-m_{1}-m_{2}\right)\right]
\end{aligned}
$$

which is proportional to $V_{2}(a)$ of the $U(N) \mathcal{N}=4$ SYM. Thus, the asymptotic behavior of the index is

$$
\left.\log I_{S^{1} \times M_{3}}\right|_{\text {orbifold SYM }}=\left.K \log I_{S^{1} \times M_{3}}\right|_{\mathcal{N}=4 \text { SYM }} .
$$

This result has a nice interpretation from the viewpoint of so-called large- $N$ orbifold equivalence [35], which states that a free energy of a "daughter" theory obtained by a projection of a "parent" theory by a group $\Gamma$ obeys

$$
\lim _{N \rightarrow \infty} \frac{F_{\text {daughter }}}{N^{2}}=\frac{1}{|\Gamma|} \lim _{N \rightarrow \infty} \frac{F_{\text {parent }}}{N^{2}},
$$

where $|\Gamma|$ is the order of $\Gamma$. Since the orbifold $\mathcal{N}=4$ SYM is obtained by a $\mathbb{Z}_{K}$ projection of the $U(K N) \mathcal{N}=4$ SYM, the above result is expected from the orbifold equivalence. For the case of the superconformal index, the entropy after the Legendre transformation is given by

\footnotetext{
${ }^{17}$ This type of ansatz was also taken in the large- $N$ analysis of the orbifold $\mathcal{N}=4$ SYM on $S^{4}$ [34].
} 
$S_{\mathrm{QFT}}(Q, J)=2 \pi \sqrt{Q_{1} Q_{2}+Q_{1} Q_{3}+Q_{2} Q_{3}-\frac{K N^{2}}{2}\left(J_{1}+J_{2}\right)}$,

which is also expressed as

$S_{\mathrm{QFT}}(Q, J)=2 \pi \sqrt{Q_{1} Q_{2}+Q_{1} Q_{3}+Q_{2} Q_{3}-2 c\left(J_{1}+J_{2}\right)}$,

via $c=K N^{2} / 2$.

\section{FUTURE DIRECTIONS}

There are several questions and interesting future directions. Perhaps the most immediate question is whether or not our results match at the quantum level. The first step to test this would be to compute a logarithmic correction to the black hole entropy by a one-loop analysis of the supergravity as in the case of the magnetically charged $\mathrm{AdS}_{4}$ black holes [36]. Our result suggests that the logarithmic correction is absent in the Cardy limit. Another question is what are physical interpretations of the dominant saddle points in the regime $\operatorname{Re}\left(\frac{i}{\tau \sigma}\right)>0$, which we have not mainly considered in this paper. The dominant saddle points in this regime are not isolated and technically give the plateau in the function $f(x)$ given in Eq. (2.10), but they are not degenerate at $\mathcal{O}\left(\beta^{-1}\right)$. This question might be related to hairy black holes [31]. It would be also interesting to study higher-order corrections of $\beta$ to the Cardy limit in order to interpolate our result to the one in Ref. [22], which does not take the Cardy limit. The higher-order corrections might be significantly different between large $N$ and finite $N$.

\section{ACKNOWLEDGMENTS}

The author thanks Nikolay Bobev, Seyed Morteza Hosseini, Seok Kim, and Leopoldo A. Pando Zayas for comments on the earlier versions of arXiv. This work has been partially supported by STFC consolidated Grant No. ST/P000681/1.

APPENDIX: EXPLICIT FORMS OF $V_{2}(a), V_{1}(a)$, AND $\tilde{V}_{1}(a)$ FOR GENERAL LAGRANGIAN 4D $\mathcal{N}=1$ THEORY

Let us consider the $4 \mathrm{~d} \mathcal{N}=1$ SUSY gauge theory with gauge group $G$ coupled to chiral multiplets of representation $\mathbf{R}_{I}$ having $U(1)_{R}$ charge $R_{I}$ and flavor charge $Q_{I}^{j}$ of $U(1)_{j}$ flavor symmetry. The refined Cardy formula takes the form [24]

$$
I_{S^{1} \times M_{3}} \underset{\beta \rightarrow 0}{\simeq} e^{-\left[\pi^{2} \operatorname{Tr}(R) L_{M_{3}} / 12 \beta\right]} \int d^{\operatorname{rank} G} a e^{-\left(\pi^{3} i A_{M_{3}} / 6 \beta^{2}\right) V_{2}(a)+\left(\pi^{2} L_{M_{3}} / 2 \beta\right) V_{1}(a)-(1 / 2 \beta) \tilde{V}_{1}(a)},
$$

where $^{18}$

$$
\begin{aligned}
& V_{2}(a)=-\sum_{\mathrm{I} \in \text { matter }} \sum_{\rho_{I} \in \mathbf{R}_{I}} \kappa\left(\rho_{I} \cdot a+\sum_{j \in \text { flavor }} Q_{I}^{j} m_{j}\right), \\
& V_{1}(a)=\sum_{\alpha \in \text { root }} \theta(\alpha \cdot a)+\sum_{\mathrm{I} \in \text { matter }} \sum_{\rho_{I} \in \mathbf{R}_{I}}\left(R_{I}-1\right) \theta\left(\rho_{I} \cdot a+\sum_{j \in \text { flavor }} Q_{I}^{j} m_{j}\right), \\
& \tilde{V}_{1}(a)=\sum_{\mathrm{I} \in \text { matter }} \sum_{\rho_{I} \in \mathbf{R}_{I}} \rho_{I} \cdot \ell_{M_{3}} \theta\left(\rho_{I} \cdot a+\sum_{j \in \text { flavor }} Q_{I}^{j} m_{j}\right) .
\end{aligned}
$$

\footnotetext{
${ }^{18}$ More generally, $\tilde{V}_{1}(a)$ can have $\ell_{M_{3}}$ for a flavor symmetry background. For example, $\ell_{M_{3}}$ for $M_{3}=S^{1} \times \Sigma_{g}$ is proportional to the magnetic charge, and we have to specify the background magnetic charges.
}

[1] J. D. Bekenstein, Black holes and the second law, Lett. Nuovo Cimento 4, 737 (1972); Black holes and entropy, Phys. Rev. D 7, 2333 (1973); Generalized second law of thermodynamics in black hole physics, Phys. Rev. D 9, 3292 (1974); S. W. Hawking, Particle creation by black holes,
Commun. Math. Phys. 43, 199 (1975); Black hole explosions, Nature (London) 248, 30 (1974).

[2] A. Strominger and C. Vafa, Microscopic origin of the Bekenstein-Hawking entropy, Phys. Lett. B 379, 99 (1996). 
[3] J. M. Maldacena, The large N limit of superconformal field theories and supergravity, Int. J. Theor. Phys. 38, 1113 (1999); Math. Phys. Appl. Math. 2, 231 (1998); S. S. Gubser, I. R. Klebanov, and A. M. Polyakov, Gauge theory correlators from noncritical string theory, Phys. Lett. B 428, 105 (1998); E. Witten, Anti-de Sitter space and holography, Adv. Theor. Math. Phys. 2, 253 (1998).

[4] F. Benini, K. Hristov, and A. Zaffaroni, Black hole microstates in $\mathrm{AdS}_{4}$ from supersymmetric localization, J. High Energy Phys. 05 (2016) 054.

[5] F. Benini, K. Hristov, and A. Zaffaroni, Exact microstate counting for dyonic black holes in AdS4, Phys. Lett. B 771, 462 (2017).

[6] F. Benini and A. Zaffaroni, A topologically twisted index for three-dimensional supersymmetric theories, J. High Energy Phys. 07 (2015) 127; Supersymmetric partition functions on Riemann surfaces, Proc. Symp. Pure Math. 96, 13 (2017); C. Closset and H. Kim, Comments on twisted indices in $3 \mathrm{~d}$ supersymmetric gauge theories, J. High Energy Phys. 08 (2016) 059; M. Honda and Y. Yoshida, Supersymmetric index on $T^{2} x S^{2}$ and elliptic genus, arXiv:1504.04355.

[7] S. M. Hosseini and A. Zaffaroni, Large $N$ matrix models for $3 \mathrm{~d} \mathcal{N}=2$ theories: Twisted index, free energy and black holes, J. High Energy Phys. 08 (2016) 064; S. M. Hosseini, A. Nedelin, and A. Zaffaroni, The Cardy limit of the topologically twisted index and black strings in $\mathrm{AdS}_{5}$, J. High Energy Phys. 04 (2017) 014; A. Cabo-Bizet, V. I. Giraldo-Rivera, and L. A. Pando Zayas, Microstate counting of $\mathrm{AdS}_{4}$ hyperbolic black hole entropy via the topologically twisted index, J. High Energy Phys. 08 (2017) 023; F. Azzurli, N. Bobev, P. M. Crichigno, V.S. Min, and A. Zaffaroni, A universal counting of black hole microstates in $\mathrm{AdS}_{4}$, J. High Energy Phys. 02 (2018) 054; S. M. Hosseini, K. Hristov, and A. Passias, Holographic microstate counting for $\mathrm{AdS}_{4}$ black holes in massive IIA supergravity, J. High Energy Phys. 10 (2017) 190; F. Benini, H. Khachatryan, and P. Milan, Black hole entropy in massive type IIA, Classical Quantum Gravity 35, 035004 (2018); N. Halmagyi and S. Lal, On the on-shell: The action of $\mathrm{AdS}_{4}$ black holes, J. High Energy Phys. 03 (2018) 146; N. Bobev, V. S. Min, and K. Pilch, Mass-deformed ABJM and black holes in $\mathrm{AdS}_{4}$, J. High Energy Phys. 03 (2018) 050; S. M. Hosseini, I. Yaakov, and A. Zaffaroni, Topologically twisted indices in five dimensions and holography, J. High Energy Phys. 11 (2018) 119; P. M. Crichigno, D. Jain, and B. Willett, 5d partition functions with a twist, J. High Energy Phys. 11 (2018) 058; M. Suh, Supersymmetric $\mathrm{AdS}_{6}$ black holes from F(4) gauged supergravity, J. High Energy Phys. 01 (2019) 035; S. M. Hosseini, K. Hristov, A. Passias, and A. Zaffaroni, 6D attractors and black hole microstates, arXiv:1809.10685; J. High Energy Phys. 12 (2018) 001; M. Suh, D4-branes wrapped on supersymmetric four-cycles from matter coupled $F(4)$ gauged supergravity, arXiv:1810.00675; On-shell action and the Bekenstein-Hawking entropy of supersymmetric black holes in $\mathrm{AdS}_{6}$, arXiv:1812.10491.

[8] J. B. Gutowski and H. S. Reall, Supersymmetric AdS(5) black holes, J. High Energy Phys. 02 (2004) 006; General supersymmetric AdS(5) black holes, J. High Energy Phys. 04 (2004) 048; Z. W. Chong, M. Cvetic, H. Lu, and C. N. Pope, Five-dimensional gauged supergravity black holes with independent rotation parameters, Phys. Rev. D 72, 041901 (2005); General Non-Extremal Rotating Black Holes in Minimal Five-Dimensional Gauged Supergravity, Phys. Rev. Lett. 95, 161301 (2005); H. K. Kunduri, J. Lucietti, and H.S. Reall, Supersymmetric multi-charge AdS(5) black holes, J. High Energy Phys. 04 (2006) 036.

[9] E. O. Colgain, M. M. Sheikh-Jabbari, J. F. Vazquez-Poritz, H. Yavartanoo, and Z. Zhang, Warped Ricci-flat reductions, Phys. Rev. D 90, 045013 (2014).

[10] M. Cvetic, M. J. Duff, P. Hoxha, J. T. Liu, H. Lu, J. X. Lu, R. Martinez-Acosta, C. N. Pope, H. Sati, and T. A. Tran, Embedding AdS black holes in ten-dimensions and eleven-dimensions, Nucl. Phys. B558, 96 (1999).

[11] S. Kim and K.-M. Lee, 1/16-BPS black holes and Giant gravitons in the $\operatorname{AdS}(5)$ X $S^{* *} 5$ space, J. High Energy Phys. 12 (2006) 077.

[12] J. Kinney, J. M. Maldacena, S. Minwalla, and S. Raju, An index for 4 dimensional super conformal theories, Commun. Math. Phys. 275, 209 (2007).

[13] C. Romelsberger, Counting chiral primaries in $\mathrm{N}=1, d=4$ superconformal field theories, Nucl. Phys. B747, 329 (2006).

[14] M. Berkooz, D. Reichmann, and J. Simon, A fermi surface model for large supersymmetric AdS(5) black holes, J. High Energy Phys. 01 (2007) 048; R. A. Janik and M. Trzetrzelewski, Supergravitons from one loop perturbative $N=4$ SYM, Phys. Rev. D 77, 085024 (2008); L. Grant, P. A. Grassi, S. Kim, and S. Minwalla, Comments on 1/16 BPS quantum states and classical configurations, J. High Energy Phys. 05 (2008) 049; M. Berkooz and D. Reichmann, Weakly renormalized near 1/16 SUSY fermi liquid operators in N = 4 SYM, J. High Energy Phys. 10 (2008) 084; C.-M. Chang and X. Yin, $1 / 16$ BPS states in $\mathcal{N}=4$ superYang-Mills theory, Phys. Rev. D 88, 106005 (2013).

[15] A. Cabo-Bizet, D. Cassani, D. Martelli, and S. Murthy, Microscopic origin of the Bekenstein-Hawking entropy of supersymmetric $\mathrm{AdS}_{5}$ black holes, arXiv:1810.11442.

[16] B. Assel, D. Cassani, and D. Martelli, Localization on Hopf surfaces, J. High Energy Phys. 08 (2014) 123.

[17] B. Assel, D. Cassani, L. Di Pietro, Z. Komargodski, J. Lorenzen, and D. Martelli, The Casimir energy in curved space and its supersymmetric counterpart, J. High Energy Phys. 07 (2015) 043.

[18] S. M. Hosseini, K. Hristov, and A. Zaffaroni, An extremization principle for the entropy of rotating BPS black holes in $\mathrm{AdS}_{5}$, J. High Energy Phys. 07 (2017) 106.

[19] N. Bobev, M. Bullimore, and H. C. Kim, Supersymmetric Casimir energy and the anomaly polynomial, J. High Energy Phys. 09 (2015) 142.

[20] S. Choi, J. Kim, S. Kim, and J. Nahmgoong, Large AdS black holes from QFT, arXiv:1810.12067.

[21] S. Choi, J. Kim, S. Kim, and J. Nahmgoong, Comments on deconfinement in AdS/CFT, arXiv:1811.08646.

[22] F. Benini and P. Milan, Black holes in $4 \mathrm{~d} \mathcal{N}=4$ SuperYang-Mills, arXiv:1812.09613.

[23] F. Benini and P. Milan, A Bethe Ansatz type formula for the superconformal index, arXiv:1811.04107; C. Closset, H. Kim, and B. Willett, $\mathcal{N}=1$ supersymmetric indices and the four-dimensional A-model, J. High Energy Phys. 08 (2017) 090. 
[24] L. Di Pietro and M. Honda, Cardy formula for 4d SUSY theories and localization, J. High Energy Phys. 04 (2017) 055.

[25] A. Arabi Ardehali, High-temperature asymptotics of supersymmetric partition functions, J. High Energy Phys. 07 (2016) 025.

[26] L. Di Pietro and Z. Komargodski, Cardy formulae for SUSY theories in $d=4$ and $d=6$, J. High Energy Phys. 12 (2014) 031.

[27] S. M. Hosseini, Black hole microstates and supersymmetric localization, arXiv:1803.01863.

[28] A. Gadde, L. Rastelli, S. S. Razamat, and W. Yan, Gauge theories and Macdonald polynomials, Commun. Math. Phys. 319, 147 (2013).

[29] T. T. Dumitrescu, G. Festuccia, and N. Seiberg, Exploring curved superspace, J. High Energy Phys. 08 (2012) 141.

[30] O. Aharony, S. S. Razamat, N. Seiberg, and B. Willett, 3d dualities from 4d dualities, J. High Energy Phys. 07 (2013) 149; A. Arabi Ardehali, J. T. Liu, and P. Szepietowski, The spectrum of IIB supergravity on $\mathrm{AdS}_{5} \times S^{5} / Z_{3}$ and a $1 / N^{2}$ test of AdS/CFT, J. High Energy Phys. 06 (2013) 024; $1 / N^{2}$ corrections to the holographic Weyl anomaly, J. High Energy Phys. 01 (2014) 002; S. Golkar and D. T. Son, (Non)-renormalization of the chiral vortical effect coefficient, J. High Energy Phys. 02 (2015) 169; A. A. Ardehali, J. T. Liu, and P. Szepietowski, $c-a$ from the $\mathcal{N}=1$ superconformal index, J. High Energy Phys. 12 (2014) 145; Central Charges from the $\mathcal{N}=1$ Superconformal Index, Phys. Rev. Lett. 114, 091603 (2015); High-temperature expansion of supersymmetric partition functions, J. High Energy Phys. 07 (2015) 113; E. Shaghoulian, Modular forms and a generalized Cardy formula in higher dimensions, Phys. Rev. D 93, 126005 (2016); Black hole microstates in AdS, Phys. Rev. D 94, 104044 (2016); M. Buican and T. Nishinaka, On the superconformal index of Argyres-Douglas theories, J. Phys. A 49, 015401 (2016).

[31] S. Bhattacharyya, S. Minwalla, and K. Papadodimas, Small hairy black holes in $\operatorname{AdS}_{5} x S^{5}$, J. High Energy Phys. 11 (2011) 035; O. J. C. Dias, P. Figueras, S. Minwalla, P. Mitra, R. Monteiro, and J. E. Santos, Hairy black holes and solitons in global $\mathrm{AdS}_{5}$, J. High Energy Phys. 08 (2012) 117; J. Markeviciute and J.E. Santos, Hairy black holes in $\mathrm{AdS}_{5} \times \mathrm{S}^{5}$, J. High Energy Phys. 06 (2016) 096; Evidence for the existence of a novel class of supersymmetric black holes with $\mathrm{AdS}_{5} \times \mathrm{S}^{5}$ asymptotics, Classical Quantum Gravity 36, 02LT01 (2019); J. Markeviciute, Rotating hairy black holes in $\mathrm{AdS}_{5} \times \mathrm{S}^{5}$, J. High Energy Phys. 03 (2019) 110.

[32] S. M. Hosseini, K. Hristov, and A. Zaffaroni, A note on the entropy of rotating BPS $\mathrm{AdS}_{7} \times S^{4}$ black holes, J. High Energy Phys. 05 (2018) 121.

[33] A. Sen, Black hole entropy function and the attractor mechanism in higher derivative gravity, J. High Energy Phys. 09 (2005) 038.

[34] T. Azeyanagi, M. Hanada, M. Honda, Y. Matsuo, and S. Shiba, A new look at instantons and large-N limit, J. High Energy Phys. 05 (2014) 008.

[35] S. Kachru and E. Silverstein, 4-D Conformal Theories and Strings on Orbifolds, Phys. Rev. Lett. 80, 4855 (1998); M. Bershadsky and A. Johansen, Large N limit of orbifold field theories, Nucl. Phys. B536, 141 (1998); P. Kovtun, M. Unsal, and L. G. Yaffe, Necessary and sufficient conditions for non-perturbative equivalences of large N(c) orbifold gauge theories, J. High Energy Phys. 07 (2005) 008.

[36] J. T. Liu, L. A. Pando Zayas, V. Rathee, and W. Zhao, OneLoop Test of Quantum Black Holes in Anti-de Sitter Space, Phys. Rev. Lett. 120, 221602 (2018). 